\title{
Profile of bavituximab and its potential in the treatment of non-small-cell lung cancer
}

This article was published in the following Dove Press journal:

Lung Cancer:Targets and Therapy

28 August 2014

Number of times this article has been viewed

Irene Stasi

\section{Federico Cappuzzo}

Medical Oncology Department, Istituto Toscano Tumori, Ospedale Civile, Livorno, Italy

Video abstract

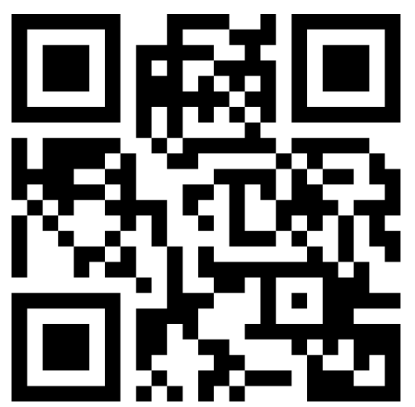

Point your SmartPhone at the code above. If you have a QR code reader the video abstract will appear. Or use: http://dvpr.es//glrgTx
Correspondence: Federico Cappuzzo Istituto Toscano Tumori, Ospedale

Civile, Viale Alfieri 36, 57I00,

Livorno, Italy

Tel +39058 6223189

$\mathrm{Fax}+390586223457$

Email f.cappuzzo@gmail.com
Abstract: Bavituximab is a an unconjugated, chimeric immunoglobulin G1 (IgG1) monoclonal antibody directed against the phosphatidylserine (PS) expressed on tumor endothelium, with a specific mechanism of action. PS is an anionic membrane phospholipid, physiologically restricted to the internal membrane leaflet; various pathophysiologic processes cause the exposure of PS on the external membrane leaflet. Bavituximab, once bound, starts up host effector activities, such as antibody dependent cellular cytotoxicity, causing vessel destruction and enhancing antitumor immunity. Phase I clinical trials of bavituximab administered as monotherapy or in combination with other chemotherapeutic agents in adults with pretreated solid tumors have been accomplished, indicating that bavituximab can be safely dispensed weekly, with a recommended dose of 1 and $3 \mathrm{mg} / \mathrm{kg}$. A Phase II randomized, placebo-controlled trial of bavituximab plus docetaxel, in the second-line therapy setting of locally advanced or metastatic non-small-cell lung cancer, has been conducted and recently presented, suggesting a clinical benefit of the combination, with an overall response rate of $17 \%$ and median overall survival of more than 11 months. A Phase III trial is currently ongoing. Bavituximab has been studied in combination with platinum-based doublets with promising results. In the present paper we summarize the preclinical development and clinical experience with bavituximab in non-small-cell lung cancer.

Keywords: bavituximab, NSCLC, targeted therapy, vascular disrupting agent, vascular targeting agent

\section{Introduction}

Metastatic non-small-cell lung cancer (NSCLC) is an invariably lethal disease with poor prognosis and with a 5-year survival rate below 5\%. ${ }^{1}$ The standard first-line therapy for advanced NSCLC not characterized for epidermal growth factor receptor (EGFR) or anaplastic lymphoma kinase (ALK) translocation is represented by platinum-based combination therapy, which prolongs survival, controls symptoms, and improves quality of life versus best supportive care in patients with good performance status. ${ }^{2}$ First-line treatment with an EGFR tyrosine kinase inhibitor (TKI), such as erlotinib hydrochloride (Tarceva $^{\circledR}$; Hoffmann-La Roche Ltd, Basel, Switzerland), gefitinib (Iressa ${ }^{\circledR}$; AstraZeneca, London, UK) or afatinib (Gilotrif ${ }^{\circledR}$; Boehringer Ingelheim GmbH, Ingelheim, Germany), is recommended in patients harboring activating EGFR mutations. Patients with ALK rearrangements are candidates for a therapy with crizotinib (Xalkori ${ }^{\circledR}$; Pfizer, New York, NY, USA), a dual mesenchymal-epithelial transition factor (MET) and ALK inhibitor. Unfortunately, irrespective of tumor biology and therapy, all patients inevitably develop resistance to any available therapy, with a median progression-free survival (PFS) of 6 months with platinum-based chemotherapy and 8-12 months for 
targeted therapies. ${ }^{2,3}$ Few effective second-line treatment options are currently available, including pemetrexed in patients with non-squamous histology, and docetaxel or erlotinib, irrespective of histology or EGFR status. ${ }^{4}$ The effect on survival of second-line therapies is generally poor, with the only exception of EGFR-TKIs in patients with EGFR mutations, or crizotinib in individuals with ALK rearrangement only in patients in which these agents were not used as front-line therapy. ${ }^{5-8}$ Hence, there is an urgent need for new, effective, and well-tolerated therapies for NSCLC, particularly in the second-line setting.

In addition to the targeted therapies mentioned above, recent insight into the molecular biology of cancer and mechanisms of tumorigenesis suggested the angiogenesis as a promising target for NSCLC treatment. The development of inhibitors of angiogenesis, also known as vessel-directed cancer treatments, has become, over the last few years, a major challenge. There are two different types of vesseldirected cancer treatments: the vascular targeting agents and the vascular disrupting agents.

The first group belong the antiangiogenic drugs, such as the anti-vascular endothelial growth factor (VEGF) monoclonal antibody bevacizumab (Avastin ${ }^{\circledR}$; GenentechRoche, South San Francisco, CA, USA), and the VEGF receptor (VEGFR) tyrosine kinase inhibitors, such as sunitinib (Sutent ${ }^{\circledR}$; Pfizer, New York, NY, USA) and sorafenib (Nexavar ${ }^{\circledR}$; Bayer HealthCare Pharmaceuticals, Leverkusen, Germany). The vascular targeting agents inhibit the formation of additional vessel from existing vasculature, mainly at the outer edge of a growing tumor, and also normalize the constitutively complex network of tumor vasculature, potentially improving the delivery and efficacy of chemotherapy. ${ }^{9}$ Bevacizumab, in combination with platinum-based chemotherapy, has been shown to improve survival versus chemotherapy alone in a first-line setting in patients with non-squamous NSCLC ${ }^{10,11}$ and currently is the only antiangiogenetic drug approved by several regulatory agencies.

In contrast, vascular disrupting agents (VDAs) are directed against existing vessels, mainly in the tumor center, ${ }^{12}$ enhancing the cytotoxicity of chemotherapy and ionizing radiation, of which efficacy is decreased by interstitial pressure and hypoxia.

Although currently under worldwide clinical investigation, these drugs are not approved for cancer treatment. ${ }^{13}$ VDAs can furthermore be divided into two classes: small molecules and ligand directed drugs. Small molecules act as microtubule destabilizers and cytokine inducers, while ligand-directed drugs target specific markers of malignant vasculature. This latest group includes bavituximab.

\section{Bavituximab}

Bavituximab is a vascular disrupting agent with a unique mechanism of action resulting in a high degree of specificity for tumor blood vessels. ${ }^{14}$ Specifically, bavituximab is an unconjugated, chimeric immunoglobulin G1 (IgG1) monoclonal antibody directed against the phosphatidylserine (PS), an anionic membrane phospholipid expressed on tumor endothelium.

Physiologically, PS is present in the membranes of all eukaryotic cells and involved in signalling, mainly in an extracellular context such as during apoptosis. PS is not evenly distributed throughout all cellular membranes, nor equally distributed between leaflets of a membrane bilayer. In healthy cells PS is restricted to the inner membrane leaflet, thanks to adenosine triphosphate (ATP)-dependent aminophospholipid translocases that catalyze the transfer from the external to the internal plasma membrane. ${ }^{15}$ Several pathophysiologic processes, such as apoptosis, cell activation, and transformation, determine the external exhibition of PS, ${ }^{16}$ due to aminophospholipid translocases inactivation, or scramblases and ABC floppases activation. ${ }^{17}$ Once external, PS acts as one major "eat me" signal, ensuring efficient recognition and uptake of apoptotic cells by phagocytes and promoting the secretion of such anti-inflammatory cytokines as interleukin-10 and transforming growth factor beta (TGF- $\beta$ ). Hypoxia and acidity, typical tumor characteristics, also trigger these processes due to the generation of the reactive oxygen species. Subsequently, PS exposure could be considered a feature of cancer cells and tumor vessel endothelium. ${ }^{18}$ Because of their large size and hydrophilic nature, monoclonal antibodies such as bavituximab do not pass through the plasma membrane and are unable to recognize and bind intracellular targets, an event that is possible once PS is external. Specifically, bavituximab binds PS when recognizing a complex formed by PS and two cross-linked molecules of the PS-binding protein beta 2 glycoprotein-1 (beta 2GP1). ${ }^{19}$

\section{Pharmacodynamics}

Bavituximab, once bound, starts up host effector activities such as antibody dependent cellular cytotoxicity, causing vessel destruction and enhancing antitumor immunity. Relevant preclinical studies have been conducted with 3G4, a mouse immunoglobulin G3 monoclonal antibody similar to bavituximab that specifically recognizes 
anionic phospholipids. ${ }^{17}$ Histologic examination of orthotopic human MDA-MB-231 tumors from mice treated with 3G4 revealed a significant reduction of neoplastic vascular density and plasma content. ${ }^{20}$ Localization of $3 \mathrm{G} 4$ to tumor vessels preceded macrophage binding to tumor vessels, impairment of vascular function, and development of necrosis. The central necrosis with a peripheral circle of surviving tumor cells is a characteristic pattern of tumor cell killing by vascular targeting agents. Indeed, vascular targeting agents are thought to be most effective against central vessels, where the high interstitial pressure contributes to vascular collapse, contrary to many anticancer therapies which are effective in the welloxygenated and peripheral areas of the tumor. Combining vascular targeting agents with antiproliferative antitumor therapies could thus have an additive or even synergistic antitumor activity.

Moreover, histologic examination revealed a marked presence of blood monocytes to tumor vascular endothelium and profuse infiltration of macrophages into the tumor interstitium, consistent with the fact that $3 \mathrm{G} 4$ enhances the rate of phagocytosis of phosphatidylserine-expressing cells by bone-marrow derived mouse macrophages in a fragment crystallizible (Fc)-dependent fashion. ${ }^{21}$ Taken together, these aspects could raise the possibility that $3 \mathrm{G} 4$ causes macrophage cytotoxicity against tumor vessels or tumor cells in mice indirectly, suggesting that the antibody is not directly cytotoxic. Two mechanisms of macrophage-mediated damage to tumor vessels or tumor cells seem feasible. Firstly, 3G4 may bind to exposed anionic phospholipids on tumor vessels and tumor cells, stimulating the binding of monocytes and macrophages via fragment crystallizible gamma (Fc-g) receptors and enhancing antibody dependent cell-mediated cytotoxicity. Secondly, 3G4 may block phosphatidylserinemediated "quiescence" signals, inducing macrophages to secrete tumor necrosis factor- $\alpha$, interleukin- 1 , and other inflammatory cytokines that directly damage tumor endothelium and recruit further host cells into the tumor. ${ }^{20}$

In addition, bavituximab provides anticancer effects by reversing the immune and inflammatory suppression exerted by tumors. If exposed, PS reduces the production of proinflammatory cytokines while PS targeting counteracts these processes, promoting proinflammatory pathways, recruitment of tumoricidal M1 macrophages, dendritic cell maturation, and antigen presentation. ${ }^{17}$

As PS performs a crucial role in the binding and activation of coagulation factors, a careful screening for an increased bleeding or thrombogenic risk has been conducted from the first studies. Unlike the pathologic antiphospholipid autoantibodies directed against cardiolipin and beta 2 glycoprotein-1 (beta 2GP1), bavituximab targets domain II of beta 2GP1, preserving its circulating and inactive conformation not correlated with antiphospholipid antibody syndrome in preclinical models. ${ }^{22}$ However, bavituximab prolongs activated partial thromboplastin time (aPTT) assays in vitro due to the binding to the phospholipid activator in the assay. Transient prolongations in the aPTT were evidenced in rats at bavituximab doses $\geq 20 \mathrm{mg} / \mathrm{kg}$ (corresponding approximately to a maximum concentration [Cmax] of $345 \mu \mathrm{g} / \mathrm{mL}$ ). Based on these results in the first Phase I trial of bavituximab, conducted on 26 pretreated cancer patients, dose escalation was capped at $3 \mathrm{mg} / \mathrm{kg}$ (simulated Cmax in humans $=65.4 \mu \mathrm{g} / \mathrm{mL}$ ). ${ }^{23}$ All six patients treated at the $3 \mathrm{mg} / \mathrm{kg}$ dose experienced a transient aPTT prolongation and none in lower dose levels. In all patients, the aPTT normalized by day three following the bavituximab dosing, consistent with the half-life of bavituximab.

An increase in d-dimer level was evidenced in about half the patients, not temporally associated with the drug administration and not predicting thrombotic occurrences. Twenty-five patients (96\%) had assessment of platelet function by platelet function analyzer 100 (PFA-100) analysis. A prolongation of normal baseline PFA-100 after bavituximab administration occurred in one patient in the $3 \mathrm{mg} / \mathrm{kg}$ dose cohort. There were no clinical or laboratory adverse events associated with this finding. Platelet activation markers, as PAC-1 and P-selectin, were unchanged in response to bavituximab therapy in the two patients evaluated.

\section{Pharmacokinetics}

The pharmacokinetics of bavituximab have been assessed in a recently published Phase I open-label, dose-escalation trial. ${ }^{23}$ The dose levels selected were $0.1,0.3,1$, and $3 \mathrm{mg} / \mathrm{kg}$, with a starting dose defined on the basis of single- and repeat-dose rodent and primate toxicity studies, and efficacy data obtained in a tumor bearing mouse model. Dose escalation to the biologically effective dose rather than the maximum tolerated dose (MTD) was the goal of this study. This approach takes into account that for monoclonal antibodies the MTD may not correspond to optimal efficacy and, in some instances, may never be reached in monotherapy studies. ${ }^{24}$

In animal models, maximal efficacy of anti-PS antibody was achieved at doses of $0.5 \mathrm{mg} / \mathrm{kg} 3$ times weekly, yielding a Cmax of $5.5 \mathrm{mg} / \mathrm{mL}$ with a half-life of 48 hours and a simulated average blood concentration of $2 \mathrm{mg} / \mathrm{mL}$. Overcoming this dose, PS binding was presumably saturated, in line with the in vitro evidence of PS-expressing cell saturation at 
1 to $2 \mathrm{mg} / \mathrm{mL}$ bavituximab concentration. Moreover, aPTT transient elevations were noted in rats at bavituximab doses of $20 \mathrm{mg} / \mathrm{kg}$ or more (Cmax $345 \mathrm{mg} / \mathrm{mL}$ ). Therefore, capping the dose escalation at $3 \mathrm{mg} / \mathrm{kg}$ (with simulated Cmax in humans of $65.4 \mathrm{mg} / \mathrm{mL}$, corresponding to an equivalent Cmax of $11-111 \mathrm{mg} / \mathrm{mL}$ in rodents) guaranteed a more than 5-fold safety value, providing drug concentrations over the therapeutic range.

Accordingly with the defined dosing schedules, bavituximab was infused to patients in the first two dose cohorts ( 0.1 and $0.3 \mathrm{mg} / \mathrm{kg}$ ) on day 0 , followed by a 28 -day washout, and then once weekly for three weeks (on days 28, 35, and 42), following patients until day $70 .{ }^{23}$ The study was subsequently amended avoiding the 28-day washout. Thus, patients in the 1 and $3 \mathrm{mg} / \mathrm{kg}$ dose cohorts have been treated with bavituximab on days $0,7,14$, and 21 and then followed until day 56. Six patients were accrued to each dose level. Dose escalation was permitted if dose-limiting toxicity (DLT) occurred in one or none of six patients and serum bavituximab levels did not exceed a predetermined threshold. Intrapatient dose escalation was not allowed. The MTD was defined as the highest dose level at which a DLT was experienced in less than 33\% of patients. DLTs were defined as any of the following adverse events observed through day 56 or 70 (depending upon cohort): grade 3 or greater hematologic or non hematologic toxicity, grade 2 or greater prolongation of prothrombin time/ international normalized ratio (PT/INR; $>1.5$ upper limit of normal [ULN] to $2>\mathrm{ULN})$, grade 3 or greater prolongation of aPTT (>2 ULN).

Bavituximab exhibited linear single-dose (day 0, for all cohorts) and multiple-dose (for the 1 and $3 \mathrm{mg} / \mathrm{kg}$ cohorts on day 21) pharmacokinetic characteristics. After time to peak concentration (Tmax), mean serum bavituximab concentrations seemed to decline in a monoexponential manner, with similar concentrations on days 0 and 21 within dose groups with a half-life of approximately two days, somewhat shorter than that of other chimeric (ie, $65 \%$ human and $35 \%$ mouse) antibodies such as cetuximab (half-life four days). This difference could be correlated with the clearance of b2GP1 as bavituximab's target: differently from soluble targets such as VEGF, PS is static and saturable, so bavituximab levels may not necessarily need to be maintained over a prolonged period. Whereas all patients experienced at least one adverse event, the most frequent were fatigue $(27 \%)$, nausea $(15 \%)$, aPTT prolongation (12\%), and pyrexia, rash, and dyspnea (each $8 \%$ ), mainly grade 1 or 2 and occurring with similar frequency across dose cohorts. Ten patients (38\%) showed at least one grade 3 or 4 adverse event. One patient experienced a pulmonary embolism considered related to treatment in the $3 \mathrm{mg} / \mathrm{kg}$ dose cohort, and was the only DLT observed in the study. Another patient in the $3 \mathrm{mg} / \mathrm{kg}$ dose cohort developed grade 3 hypertension considered not causally related to bavituximab. The authors concluded, therefore, that bavituximab can be safely administered weekly, with low risk of infusion reactions. On the basis of sustained achievement of predicted therapeutic concentrations, both the 1 and $3 \mathrm{mg} / \mathrm{kg}$ doses are recommended for subsequent monotherapy and combination trial with a close clinical monitoring for thromboembolic phenomena and laboratory monitoring for antiphospholipid effect, in consideration of the case of pulmonary embolism that occurred.

\section{Clinical data}

Clinical data regarding the efficacy of bavituximab was initially derived from the aforementioned Phase I study. Of the 26 patients enrolled and affected by cancer of the breast $(n=13)$, colorectal $(n=4)$, pancreas $(n=3)$, and hepatocellular, head and neck, melanoma, mesothelioma and prostate $(n=1$ each), 18 were evaluable, presenting as eight stable disease and ten progressive disease, according to revised Response Evaluation Criteria in Solid Tumors (RECIST) guideline (version 1.1) RECIST criteria. There were no objective responses, similarly to efficacy data regarding other vascular targeting monoclonal antibodies.

In subsequent Phase Ib, which enrolled 14 patients with refractory advanced solid tumors, bavituximab (B) was given weekly for up to 8 weeks, at a dose of $3 \mathrm{mg} / \mathrm{kg}$, in combination with docetaxel (D) $\left(75-80 \mathrm{mg} / \mathrm{m}^{2}\right.$ every 3 weeks) or gemcitabine $(\mathrm{G})\left(1,000 \mathrm{mg} / \mathrm{m}^{2}\right.$ in weeks $\left.1-7\right)$, or paclitaxel $\left(175 \mathrm{mg} / \mathrm{m}^{2}\right.$ every three weeks) plus carboplatin (appropriate use criteria $[\mathrm{AUC}]=5)(\mathrm{P}+\mathrm{C})$. Study objectives were to evaluate the safety, tolerability and pharmacokinetic profile of B when combined with chemotherapeutic agents. Serum levels of B were determined by enzyme-linked immunosorbent assay (ELISA). Five patients received $\mathrm{B}+\mathrm{D}$, five patients received $\mathrm{B}+\mathrm{G}$, and four patients received $\mathrm{B}+\mathrm{P}+\mathrm{C}$. The most common adverse events were vomiting (six patients), cough (four patients), asthenia, pain, headache, neutropenia, prolonged aPTT, and pain in extremities, each in three patients, with three serious adverse events (SAEs) considered related to $\mathrm{B}$. In accordance with RECIST criteria, among the twelve evaluable patients there was partial response (three patients), stable disease (three patients), and progressive disease (six patients). Regarding the pharmacokinetic profile, no significant differences were seen between the three treatment groups following administration of $\mathrm{B}$ on days 0 
and 21; no accumulation of B was seen following multiple dose administration. ${ }^{25}$

On the basis of these results (Table 1), a randomized, blinded, placebo-controlled Phase II trial of D plus B as second-line treatment in locally advanced or metastatic NSCLC patients was conducted. ${ }^{26}$ One hundred and twentyone patients were randomized 1:1:1 to receive D at $75 \mathrm{mg}$ / $\mathrm{m}^{2}$ every 21 days for up to 6 cycles in combination with blinded infusions of placebo (P), B at $1 \mathrm{mg} / \mathrm{kg}$, or B at $3 \mathrm{mg}$ / $\mathrm{kg}$ weekly, until disease progression or unacceptable toxicity. The primary endpoint was overall response rate (ORR), while secondary endpoints were progression-free survival (PFS) and overall survival (OS). After the study unblinding, a pharmacokinetic predefined analysis revealed phial coding discrepancies in the placebo and B at $1 \mathrm{mg} / \mathrm{kg}$ phials; consequently data from these two cohorts were pooled forming the control arm (C arm). Between the 41 patients treated with $\mathrm{B}$ at $3 \mathrm{mg} / \mathrm{kg}$ plus $\mathrm{D}$, representing the experimental arm, and the other 80 patients into the $\mathrm{C}$ arm, there were no significant differences in age, sex, ethnicity or disease stage. Available data, collected at $54 \%$ of death events reported in the experimental arm and $71 \%$ in the $\mathrm{C}$ arm, demonstrated $17.1 \%$ and $13.8 \%$ ORR, respectively. Regarding secondary endpoints, median PFS was 4.5 months and median OS was 11.7 months for $3 \mathrm{mg} / \mathrm{kg} \mathrm{B}+\mathrm{D}$ arm; median PFS was 3.3 months, and median OS was 7.3 months for $\mathrm{C}$ arm (Table 2). The safety profile for $3 \mathrm{mg} / \mathrm{kg} \mathrm{B}+\mathrm{D}$ was similar to that of $\mathrm{C}$ arm in severity and frequency, representing the planned dose for the Phase III trial.

The SUNRISE ("Stimulating ImmUne RespoNse thRough BavItuximab in a PhaSE III Lung Cancer Study") is the Phase III global, randomized, double-blind, placebocontrolled clinical trial, recently opening to enrollment in more than 100 clinical sites worldwide, which randomized, in 1:2 fashion, to bavituximab plus docetaxel versus docetaxel plus placebo as second line in approximately 600 patients with Stage IIIb/IV non-squamous NSCLC. All patients will receive up to six 21-day cycles of docetaxel $\left(75 \mathrm{mg} / \mathrm{m}^{2}\right)$ plus weekly infusions of either bavituximab $(3 \mathrm{mg} / \mathrm{kg})$ or placebo until progression or toxicity. The primary endpoint of the trial is OS.

In January 2014 bavituximab was granted the Fast Track designation by the US Food and Drug Administration (FDA) for the potential treatment of second-line NSCLC. The designation emphasized the great interest in this drug.

Bavituximab has also been studied in the frontline setting. In a Phase II trial ${ }^{27}$ which enrolled 49 Indian patients with untreated locally advanced or metastatic NSCLC, the antibody, at a weekly dose of $3 \mathrm{mg} / \mathrm{kg}$ until disease progression, was tested in combination with carboplatin and paclitaxel, at a dose of $\mathrm{AUC}=5$ and $175 \mathrm{mg} / \mathrm{m}^{2}$, respectively, on day 1 up to six 21-day cycles.

The study used a Simon 2-stage design and demonstrated an ORR (complete response + partial response) in the initial group of $11 / 21(52.4 \%)$, with clinical complete response in $4.8 \%$ and partial response in $47.6 \%$. According to available data, median PFS in the first 21 patients treated is 6.2 months; median duration of response and median overall survival have not yet been presented. Adverse events (AEs) have been reported by $44 / 49(89.8 \%)$ treated subjects, the most common being pyrexia (28.6\%), diarrhea (26.5\%), alopecia $(26.5 \%)$, anemia (22.4\%), neuropathy (20.4\%), and pain (20.4\%) with mild or moderate severity. Forty-five SAEs have been evidenced in 19 subjects, including two deaths due to myocardial infarctions and one death due to hemoptysis, which occurred in a subject with squamous histology, whereupon the accrual of squamous cell carcinoma ceased.

On the basis of the promising result of 52\% ORR using RECIST criteria in an initial group, the combination with carboplatin and paclitaxel has been evaluated in a recentlyconcluded Phase II randomized trial (NCT01160601), which accrued a total of 86 subjects in the United States, the Republic of India, the Republic of Georgia, the Russian Federation, and Ukraine. The trial randomized patients with up to six cycles of carboplatin $(\mathrm{AUC}=6)$ plus paclitaxel at $200 \mathrm{mg} / \mathrm{m}^{2}$ with or without bavituximab $3 \mathrm{mg} / \mathrm{kg}$ weekly until intolerable toxicities or disease progression. No data have yet been presented. Another combination,

Table I Published trials conducted in refractory advanced solid tumor patients

\begin{tabular}{|c|c|c|c|c|c|c|c|}
\hline Study & Phase & $\mathbf{N}$ & Dose & Treatment & $\begin{array}{l}\text { ORR } \\
(C R+P R+S D)\end{array}$ & PD & PFSIOS \\
\hline Gerber et $\mathrm{al}^{23}$ & I & 18 & I-3 mg/mq weekly & Monotherapy & $50 \%$ & $50 \%$ & NA \\
\hline Digumarti et al ${ }^{25}$ & $\mathrm{lb}$ & 14 & 3 mg/mq weekly & $\begin{array}{l}\text { Combination } \\
\text { with } D / G / P+C\end{array}$ & $50 \%$ & $50 \%$ & NA \\
\hline
\end{tabular}

Abbreviations: $\mathrm{N}$, number of patients; ORR, overall response rate; mq, square meter; $\mathrm{CR}$, complete response; PR, partial response; $\mathrm{SD}$, stable disease; $\mathrm{PD}$, progressive disease; PFS, progression free survival; OS, overall survival; D, docetaxel; G, gemcitabine; P + C, paclitaxel + carboplatin; NA, not available. 
Table 2 Presented trials conducted in NSCLC patients

\begin{tabular}{|c|c|c|c|c|c|c|c|}
\hline Study & Phase & $\mathbf{N}$ & Dose & Treatment & ORR (CR + PR + SD) & PFS & OS \\
\hline \multirow[t]{2}{*}{ Shtivelband et $\mathrm{al}^{26}$} & II R & 121 & I-3 mg/mq weekly & Second line, & I7. $1 \%(E)$ & $4.5 \mathrm{~m}(\mathrm{E})$ & $11.7 \mathrm{~m}(\mathrm{E})$ \\
\hline & & & & $\begin{array}{l}\text { combination with } \mathrm{D} \text {, } \\
\text { randomized to bavituximab } \\
\text { (E) or placebo }(\mathrm{C})\end{array}$ & $13.8 \%(C)$ & $3.3 \mathrm{~m}(\mathrm{C})$ & $7.3 \mathrm{~m}(\mathrm{C})$ \\
\hline Digumarti et $\mathrm{al}^{27}$ & II & 49 & 3 mg/mq weekly & $\begin{array}{l}\text { First line, } \\
\text { combination with } P+C\end{array}$ & $52.4 \%$ & $6.2 \mathrm{~m}$ & NA \\
\hline
\end{tabular}

Abbreviations: NSCLC, non-small-cell lung cancer; N, number of patients; R, randomized; mg, square meter; ORR, overall response rate; CR, complete response; PR, partial response; SD, stable disease; PFS, progression free survival; OS, overall survival; D, docetaxel; P + C, paclitaxel + carboplatin; E, experimental arm; C, control arm; NA, not available; $m$, months.

specifically carboplatin, pemetrexed and bavituximab, is studied in the same setting in a recently opened Phase I trial (NCT01323062).

\section{Discussion}

Angiogenesis, thanks to progressive insight into the molecular biology of cancer and mechanisms of tumorigenesis, is a promising target for NSCLC treatment, and the development of vessel-directed cancer treatments has grown enormously in recent years. These treatments can be divided into two different classes: the vascular targeting agents (such as bevacizumab, the only antiangiogenetic drug today approved for NSCLC treatment), and the vascular disrupting agents such as bavituximab.

Bavituximab is a an unconjugated, chimeric immunoglobulin G1 (IgG1) monoclonal antibody directed against the phosphatidylserine (PS) expressed on tumor endothelium and segregated to the inner membrane leaflet. Once exposed on the external membrane, due to various pathophysiologic processes, PS can be bound by bavituximab, which starts up host effector activities such as antibody dependent cellular cytotoxicity, resulting in vessel destruction and enhanced antitumor immunity.

On the basis of promising preclinical activity and an acceptable safety profile, bavituximab is currently under investigation in combination with chemotherapy for advanced NSCLC in several trials. The second-line setting studies are of particular clinical interest. Specifically, a recently presented Phase II randomized, placebocontrolled trial of bavituximab plus docetaxel suggested a positive trend favoring the combination, with ORR of $17 \%$, median PFS more than 4 months, and median OS more than 11 months. These results, although initial and needing confirmation by the currently ongoing Phase III trial, are promising and similar to data recently published with docetaxel plus nintedanib, an oral angiokinase inhibitor, in the same setting. ${ }^{28}$ This latest trial demonstrates a significant OS benefit, thus is different from other antiangiogenic compounds that have been tested in the second-line setting, such as vandetanib, ${ }^{29}$ sunitinib, ${ }^{30}$ or aflibercept, ${ }^{31}$ despite PFS improvements.

In view of the hypothetical association of antiphospholipid antibody syndrome with cases of thrombosis and bleeding occurring in earlier studies, current clinical studies are characterized by careful laboratory monitoring which definitively clarifies these features.

A principal limitation of available studies is the lack of biomarker data, which represents an urgent major challenge. Although in vitro aPTT prolongation provides some pharmacodynamic data, the association between this laboratory finding and therapeutic effect is not known. To provide more insight into the mechanism of bavituximab in humans, dynamic contrast enhanced magnetic resonance imaging (MRI) scans could be incorporated into selected ongoing and future bavituximab clinical trials. Similarly, the available studies did not include biomarkers of antitumor immunity, which represents another proposed mechanism of bavituximab therapeutic effect.

In conclusion, available data regarding the use of bavituximab for the treatment of metastatic and refractory NSCLC make an important contribution in cancer treatment research by adding another agent to the armamentarium of drugs. It is hoped that the use of more comprehensive study designs incorporating tissue-, blood-, and imaging-based assays will result in even more clinically relevant results.

\section{Acknowledgment}

Supported in part by Associazione Italiana ricerca cancro (AIRC), Istituto Toscano Tumori, Fondazione Ricerca Traslazionale (FoRT).

\section{Disclosure}

The authors report no conflicts of interest in this work.

\section{References}

1. American Cancer Society. Non-Small Cell Cancer Survival Rates by Stage. Available from: http://www.cancer.org/cancer/lungcancer-nonsmallcell/detailedguide/non-small-cell-lung-cancer-survival-rates. Accessed May 22, 2013. 
2. Peters S, Adjei AA, Gridelli C, Kerr K, Felip E. Metastatic non-small-cell lung cancer (NSCLC): ESMO Clinical Practice Guidelines for diagnosis, treatment and follow-up. Ann Oncol. 2012;23,Suppl7:vii56-vii64.

3. Sculier JP, Moro-Sibilot D. First- and second-line therapy for advanced nonsmall cell lung cancer. Eur Respir J. 2009;33:915-930.

4. Oxnard GR, Arcila ME, Chmielecki J, Ladanyi M, Miller VA, Pao W. New strategies in overcoming acquired resistance to epidermal growth factor receptor tyrosine kinase inhibitors in lung cancer. Clin Cancer Res. 2011;17(17):5530-5537.

5. Shepherd FA, Dancey J, Ramlau R, et al. Prospective randomized trial of docetaxel versus best supportive care in patients with non-small-cell lung cancer previously treated with platinum-based chemotherapy. J Clin Oncol. 2000;18(10):2095-2103.

6. Hanna N, Shepherd FA, Fossella FV, et al. Randomized phase III trial of pemetrexed versus docetaxel in patients with non-small-cell lung cancer previously treated with chemotherapy. J Clin Oncol. 2004;22(9): 1589-1597.

7. Shepherd FA, Rodrigues PJ, Ciuleanu T, et al. Erlotinib in previously treated non-small-cell lung cancer. $N$ Engl J Med. 2005;353: 123-132.

8. Hensing TA, Schell MJ, Lee JH, Socinski MA. Factors associated with the likelihood of receiving second line therapy for advanced non-small cell lung cancer. Lung Cancer. 2005;47(2):253-259.

9. Willett CG, Boucher Y, di Tomaso E, et al. Direct evidence that the VEGF-specific antibody bevacizumab has antivascular effects in human rectal cancer. Nat Med. 2004;10(2):145-147.

10. SoriaJC,MauguenA,ReckM,etal.Systematicreviewandmeta-analysisofrandomised, phase II/III trials adding bevacizumab to platinum-based chemotherapy as first-line treatmentinpatientswithadvancednon-small-cell lung cancer.Ann Oncol. 2013;24(1): 20-30.

11. Patel JD, Socinski MA, Garon EB, et al. PointBreak: a randomized phase III study of pemetrexed plus carboplatin and bevacizumab followed by maintenance pemetrexed and bevacizumab versus paclitaxel plus carboplatin and bevacizumab followed by maintenance bevacizumab in patients with stage IIIB or IV nonsquamous non-small-cell lung cancer. J Clin Oncol. 2013;31(34):4349-4357.

12. Martin SJ, Reutelingsperger CP, McGahon AJ, et al. Early redistribution of plasma membrane phosphatidylserine is a general feature of apoptosis regardless of the initiating stimulus: inhibition by overexpression of Bcl-2 and Abl. J Exp Med. 1995;182(5): 1545-1556.

13. Zhao J, Zhou Q, Wiedmer T, Sims PJ. Level of expression of phospholipid scramblase regulates induced movement of phosphatidylserine to the cell surface. J Biol Chem. 1998;273(12):6603-6606.

14. De Rose P, Thorpe PE, Gerber DE. Development of bavituximab, a vascular targeting agent with immune-modulating properties, for lung cancer treatment. Immunotherapy. 2011;3(8):933-944.

15. Ran S, Thorpe PE. Phosphatidylserine is a marker of tumor vasculature and a potential target for cancer imaging and therapy. Int J Radiat Oncol Biol Phys. 2002;54(5):1479-1484.

16. Luster TA, He J, Huang X, et al. Plasma protein beta-2-glycoprotein 1 mediates interaction between the anti-tumor monoclonal antibody $3 \mathrm{G} 4$ and anionic phospholipids on endothelial cells. J Biol Chem. 2006;281(40):29863-29871.

17. Ran S, He J, Huang X, Soares M, Scothorn D, Thorpe PE. Antitumor effects of a monoclonal antibody that binds anionic phospholipids on the surface of tumor blood vessels in mice. Clin Can Res. 2005;11(4):1551-1562.
18. Thorpe PE. Vascular targeting agents as cancer therapeutics. Clin Cancer Res. 2004;10(2):415-427.

19. Fadok VA, Bratton DL, Konowal A, Freed PW, Westcott JY, Henson PM. Macrophages that have ingested apoptotic cells in vitro inhibit proinflammatory cytokine production through autocrine/paracrine mechanisms involving TGF-beta, PGE2, and PAF. J Clin Invest. 1998;101(4):890-898

20. Chen X, Doffek K, Sugg SL, Shilyansky J. Phosphatidylserine regulates the maturation of human dendritic cells. J. Immunol. 2004;173(5): 2985-2994.

21. He J, Yin Y, Luster TA. Antiphosphatidylserine antibody combined with irradiation damages tumor blood vessels and induces tumor immunity in a rat model of glioblastoma. Clin Cancer Res. 2009;15(22): 6871-6880.

22. Pregnolato F, Chighizola CB, Encabo S. Anti-phosphatidylserine/ prothrombin antibodies: an additional diagnostic marker for APS? Immunol Res. 2013;56(2-3):432-438.

23. Gerber DE, Stopeck A, Wong L, et al. Phase I safety and pharmacokinetic study of bavituximab, a chimeric phosphatidylserine-targeting monoclonal antibody, in patients with advaced solid tumors. Clin Cancer Res. 2005;17(21):6888-6896.

24. Beckman RA, von Roemeling R, Scott AM. Monoclonal antibody dose determination and biodistribution into solid tumors. Ther Deliv. 2011;2(3):333-344.

25. Digumarti R, Bapsy PP, Shan JS, et al. A phase Ib safety and pharmacokinetic study of bavituximab plus chemotherapy in patients with refractory advanced solid tumor malignancies. J Clin Oncol (Meeting Abstracts). 2008;26(15)(suppl:3038).

26. Shtivelband M, Spigel DR, Gerber DE, et al. Randomized, blinded, placebo-controlled phase II trial of docetaxel and bavituximab as second-line therapy in locally advanced or metastatic non-squamous non-small cell lung cancer. J Clin Oncol (Meeting Abstracts). 2013;31(suppl:8095).

27. Digumarti R, Suresh AV, Bharracharyya GS, Dasappa L, Shan J. Phase II study of bavituximab plus paclitaxel and carboplatin in untreated locally advanced or metastatic non-small cell lung cancer: interim results. J Clin Oncol (Meeting Abstracts). 2010;28 (suppl:7589).

28. Reck, M, Kaiser, R, Mellemgaard A, et al. Docetaxel plus nintedanib versus docetaxel plus placebo in patients with previously treated non-small-cell lung cancer (LUME-Lung 1): a phase 3, double-blind, randomised controlled trial. Lancet Oncol. 2014;15(2):143-155.

29. Herbst RS, Sun Y, Eberhardt WE, et al. Vandetanib plus docetaxel versus docetaxel as second-line treatment for patients with advanced non-small-cell lung cancer (ZODIAC): a double-blind, randomised, phase 3 trial. Lancet Oncol. 2010;11(7):619-626.

30. Scagliotti GV, Krzakowski M, Szczesna A, et al. Sunitinib plus erlotinib versus placebo plus erlotinib in patients with previously treated advanced non-small-cell lung cancer: a phase III trial. J Clin Oncol. 2012;30(17):2070-2078.

31. Ramlau R, Gorbunova V, Ciuleanu TE, et al. Aflibercept and docetaxel versus docetaxel alone after platinum failure in patients with advanced or metastatic non-small-cell lung cancer: a randomized, controlled phase III trial. J Clin Oncol. 2012;30(29):3640-3647. 


\section{Publish your work in this journal}

Lung Cancer: Targets and Therapy is an international, peer-reviewed, open access journal focusing on lung cancer research, identification of therapeutic targets and the optimal use of preventative and integrated treatment interventions to achieve improved outcomes, enhanced survival and quality of life for the cancer patient. Specific topics covered in the journal include: Epidemiology, detection and screening; Cellular research and biomarkers; Identification of biotargets and agents with novel mechanisms of action; Optimal clinical use of existing anticancer agents, including combination therapies; Radiation and surgery; Palliative care; Patient adherence, quality of life, satisfaction; Health economic evaluations. The manuscript management system is completely online and includes a very quick and fair peer-review system. Visit http://www.dovepress.com/testimonials.php to read real quotes from published authors.

Submit your manuscript here: http://www.dovepress.com/lung-cancer-targets--therapy-journal 